\title{
Guest editorial: Connecting multiple aspects of hematologic malignancies toward creation of new therapeutics
}

\author{
Fumihiko Ishikawa ${ }^{1}$
}

Received: 6 February 2017 / Accepted: 9 February 2017 / Published online: 23 February 2017

(C) The Japanese Society of Hematology 2017

The field of malignant stem cell research was pioneered by acute myeloid leukemia (AML) research. Every hematologist must recall an original article in Nature, 1994, in which Dr. Dick and his colleagues found preferential engraftment in immune-compromised mice by $\mathrm{CD} 34^{+} \mathrm{CD} 38^{-} \mathrm{AML}$ cells [1]. This exciting report on phenotypic and functional heterogeneity within human AML led the way to investigations into potential heterogeneity in solid tumors as well as diverse hematologic malignancies.

The existence of pre-leukemic stem cells is another groundbreaking discovery, reported more recently [2-4]. In the pre-leukemic stem cell model, it is thought that multiple somatic mutations hit immature hematopoietic stem and progenitor cells and incite genetic events that ultimately lead to leukemogenic transformation. Moreover, following the discovery of pre-leukemic stem cells, the presence of AML-associated mutations, such as DNMT3A, TET2, and ASXL1 in the elderly population without hematologic diseases were reported by three groups [5-7]. This phenomenon is thought to be analogous to MGUS in B cell malignancies and was coined clonal hematopoiesis with indeterminate potential (CHIP) [8]. To what extent CHIP contributes to myeloid leukemogenesis is unclear and is an area of active investigation.

One of the most outstanding technical advancements over the past decade is genome sequencing technology enabling investigators to rapidly identify numerous mutations. The discovery of multiple somatic mutations in people with and without diseases has spurred efforts to understand the

Fumihiko Ishikawa

fumihiko.ishikawa@riken.jp

1 Laboratory for Human Disease Models, RIKEN Center for Integrative Medical Sciences, Yokohama, Japan functional significance of those mutations. Clarifying the roles played by these mutations, individually and in combination, may help create new and effective therapeutic options.

In this chapter, review articles by three experts introduce multiple aspects of hematologic malignancies and how they must be integrated to create novel therapeutics. Cytokine receptor signaling, leukemia-environment interactions, and leukemia stem cell-specific plasma membrane molecules, each essential for understanding biology of leukemogenesis, are presented. Each article clearly discusses the distinction between leukemic stem cells and normal hematopoietic stem cells that contribute to lifelong production and maintenance of homeostasis in blood and immune systems. Furthermore, the merits of both genetically-modified mouse models and patient-derived xenograft models are considered, presenting overlapping and distinct strengths for various research objectives.

I hope that multi-faceted analyses integrating emerging new technologies lead to new pathways to effective treatment options for patients with difficult-to-treat hematologic malignancies.

\section{References}

1. Lapidot T, Sirard C, Vormoor J, et al. A cell initiating human acute myeloid leukaemia after transplantation into SCID mice. Nature. 1994;367:645-8.

2. Jan M, Snyder TM, Corces-Zimmerman MR, et al. Clonal evolution of preleukemic hematopoietic stem cells precedes human acute myeloid leukemia. Sci Transl Med. 2012;4:149ra118.

3. Corces-Zimmerman MR, Hong WJ, Weissman IL, et al. Preleukemic mutations in human acute myeloid leukemia affect epigenetic regulators and persist in remission. Proc Natl Acad Sci USA. 2014;111:2548-53. 
4. Shlush LI, Zandi S, Mitchell A, et al. Identification of pre-leukaemic haematopoietic stem cells in acute leukaemia. Nature. 2014;506:328-33.

5. Jaiswal S, Fontanillas P, Flannick J, et al. Age-related clonal hematopoiesis associated with adverse outcomes. N Engl J Med. 2014;371:2488-98.

6. Genovese G, Kähler AK, Handsaker RE, et al. Clonal hematopoiesis and blood-cancer risk inferred from blood DNA sequence. N Engl J Med. 2014;371:2477-87.
7. Xie M, Lu C, Wang J, et al. Age-related mutations associated with clonal hematopoietic expansion and malignancies. Nat Med. 2014;20:1472-8.

8. Steensma DP, Bejar R, Jaiswal S, et al. Clonal hematopoiesis of indeterminate potential and its distinction from myelodysplastic syndromes. Blood. 2015;126:9-16. 